\title{
Modality specific neural correlates of auditory and somatic hallucinations
}

\author{
S S Shergill, L A Cameron, M J Brammer, S C R Williams, R M Murray, P K McGuire
}

\begin{abstract}
Somatic hallucinations occur in schizophrenia and other psychotic disorders, although auditory hallucinations are more common. Although the neural correlates of auditory hallucinations have been described in several neuroimaging studies, little is known of the pathophysiology of somatic hallucinations. Functional magnetic resonance imaging (fMRI) was used to compare the distribution of brain activity during somatic and auditory verbal hallucinations, occurring at different times in a 36 year old man with schizophrenia. Somatic hallucinations were associated with activation in the primary somatosensory and posterior parietal cortex, areas that normally mediate tactile perception. Auditory hallucinations were associated with activation in the middle and superior temporal cortex, areas involved in processing external speech. Hallucinations in a given modality seem to involve areas that normally process sensory information in that modality. (f Neurol Neurosurg Psychiatry 2001;71:688-690)
\end{abstract}

Keywords: functional magnetic resonance imaging; auditory hallucinations; somatic hallucinations

A perception of being touched in the absence of a sensory stimulus is termed a somatic hallucination. These occur in schizophrenia and other psychotic disorders, although auditory hallucinations are more common. Although the neural correlates of auditory hallucinations have been described in several neuroimaging studies, ${ }^{1-4}$ little is known of the pathophysiology of somatic hallucinations. An EEG study of a patient experiencing somatic hallucinations linked these with activity in the right parietal operculum, post central gyrus, and insula, ${ }^{5}$ although precise spatial localisation is difficult with this technique. The only previous neuroimaging study used single photon emission tomography to measure resting activity in chronic schizophrenic patients with somatic hallucinations. In this report activity was relatively reduced in the inferior temporal region and increased in the basal ganglia. ${ }^{6}$ In the present study, we examined the distribution of neural activity while a patient was actually experiencing somatic hallucinations, and contrasted this with activity when he was experiencing auditory hallucinations or was hallucination free.

\section{Case report}

CLINICAL HISTORY

We studied a 36 year old right handed man with a 6 year history of DSM-IV schizophrenia, ${ }^{7}$ who experienced both somatic and auditory hallucinations, often and intermittently. His somatic hallucinations involved an unpleasant perception of being "touched by spirits" predominantly in the perineum, back, and head. His auditory hallucinations involved hearing predominantly derogatory "voices" talking to him in the second and third person. $\mathrm{He}$ was being treated with olanzapine $(10 \mathrm{mg}$ daily) and provided informed consent to participate in the study.

\section{METHODS}

He was examined using a novel functional MRI (fMRI) acquisition method, which permits the measurement of spontaneous neural activity without requiring subjects to signal when hallucinations occur. ${ }^{1} \mathrm{He}$ lay in the scanner with his eyes closed. At randomly varied intervals of 30-60 seconds he heard the noise generated by the acquisition of a single volume of fMRI data (lasting 3 seconds). This served as an auditory cue for the patient to describe whatever he had been experiencing in the few seconds before the noise. Because there is a lag of 3-5 seconds between neural activity and the maximum BOLD signal ${ }^{8}$ (reflecting the local haemodynamic response to that activity), the resultant image depicts the activity that was occurring a few seconds before the onset of the cue. After providing a description, the subject returned to the resting state, during which he experienced intermittent auditory or somatic hallucinations.

IMAGE ACQUISITION AND ANALYSIS

Gradient echo echoplanar MR images were acquired using a 1.5 Tesla GE Signa system fitted with advanced NMR hardware and software. In each of 14 non-contiguous planes parallel to the intercommissural (AC-PC) plane, 40-100 T2* weighted MR images depicting BOLD contrast were acquired with $\mathrm{TE}=40$ $\mathrm{ms}, \mathrm{TR}=3000 \mathrm{~ms}$, in plane resolution=3.1 $\mathrm{mm}$, slice thickness $=7 \mathrm{~mm}$, slice $=$ skip $0.7 \mathrm{~mm}$. 
Table 1 Regions showing activation during auditory and somatic hallucinations (threshold $p<0.001$ )

\begin{tabular}{lllll}
\hline Region & $\begin{array}{l}\text { No of } \\
\text { voxels }\end{array}$ & $x$ & $y$ & $z$ \\
\hline Activation during somatic hallucinations: & & & & \\
$\quad$ Left middle frontal gyrus & 55 & -35 & 36 & 20 \\
$\quad$ Left postcentral gyrus & 44 & -29 & -19 & 31 \\
& 13 & -58 & -22 & 15 \\
Left insula & 41 & -38 & 14 & 4 \\
Left posterior cingulate gyrus & 37 & -14 & -44 & 9 \\
Right posterior cingulate gyrus & 31 & 3 & -47 & 37 \\
Right thalamus & 29 & 23 & -31 & 4 \\
Left middle temporal gyrus & 27 & -55 & -44 & 9 \\
$\quad$ Left precentral gyrus & 22 & -46 & 6 & 9 \\
Right precuneus & 15 & 23 & -72 & 26 \\
Left superior temporal gyrus & 12 & -46 & -47 & 15 \\
Right inferior frontal gyrus & 10 & 52 & 19 & 26 \\
Right insula & 7 & 35 & 22 & 4 \\
$\quad$ Left inferior parietal lobule & 4 & -55 & -39 & 31 \\
Left thalamus & 4 & -3 & -6 & 4 \\
Activation during auditory hallucinations: & & & & \\
Right superior temporal gyrus/insula & 64 & 32 & -17 & 15 \\
Right middle temporal gyrus & 16 & 35 & -58 & 26 \\
Right parahippocampal gyrus & 3 & 17 & -25 & -13 \\
\hline
\end{tabular}

*Areas of significant activation in direct comparison of somatic versus auditory hallucinations.

After the minimisation of movement related artifacts by realignment and regression ${ }^{9}$ voxelwise activation maps were constructed by computing the product moment correlation of the time series at each voxel ${ }^{10}$ with the reported occurrence of auditory hallucinations. Ten further maps were computed at each voxel after randomly permuting the pattern of auditory hallucination reports. After mapping of observed and randomised correlation data into standard stereotactic space, ${ }^{11}$ median observed and randomised maps were constructed. Foci of activation with a voxel-wise probability of type I error of $<0.0001$ (at this level of significance there should be $<1$ random error voxel per slice of data) were identified by determining the critical threshold from the distribution of correlation coefficients computed after random permutation..$^{912}$ Activation maps were overlaid on a template in standard stereotactic space.

\section{Results}

Twelve volumes were associated with reports of somatic hallucinations, 36 volumes with auditory hallucinations, and 12 with no hallucinations. Somatic hallucinations were reported as occurring on the back (three image volumes), the back of the head (one), left ear (one), right arm and back (one), right leg/foot (three) and perineum/genitals (three). The brain regions associated with the presence of somatic and auditory hallucinations are described in table 1 and shown in figure $1 \mathrm{~A}$ and $\mathrm{B}$.

The main areas activated in association with somatic hallucinations (relative to nonhallucinating events) were the thalamus, insula,

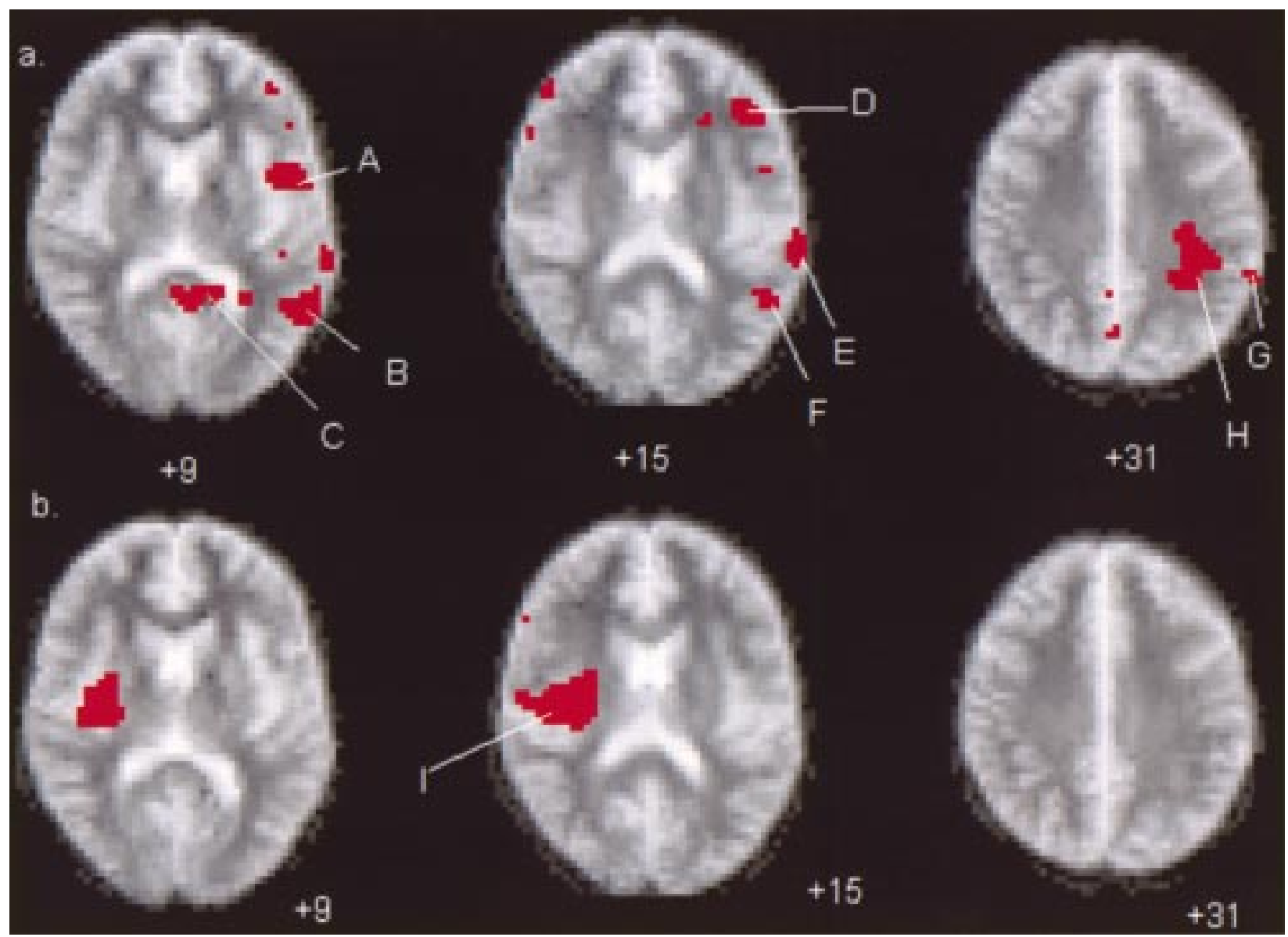

Figure 1 Ascending transverse sections through the brain from left to right, relative to the intercommissural plane (mm) illustrating the brain areas associated with row (a) somatic hallucinations and row (b) auditory hallucinations. The right side of the brain is shown in the left side of each image. The main foci of activation are shown in red: (1) During somatic hallucinations these were in the left precentral gyrus (Talairach coordinates ( $x, y, z)$ A), the left posterior middle temporal gyrus (B), the posterior cingulate gyrus bilaterally (C), the left middle frontal gyrus (D), twin foci in the postcentral gyrus $(E, H)$, the posterior superior temporal gyri $(F)$, inferior parietal lobule $(G)$, thalamus, insula bilaterally with rightsided inferior frontal gyrus, and precuneus. (2) Brain areas activated in association with auditory hallucinations were the right posterior insula/superior temporal gyrus (I). 
and posterior cingulate gyrus bilaterally. Further activation was evident in the left middle frontal gyrus, the precentral gyrus, twin foci in the postcentral gyrus, inferior parietal lobule, the middle and superior temporal gyri with right sided inferior frontal gyrus, and precuneus (fig $1 \mathrm{~A}$ ). By contrast, auditory hallucinations were associated with activation in a large region spanning the right superior temporal gyrus and posterior insula, the right middle temporal gyrus, and hippocampus/ parahippocampal gyrus (fig $1 \mathrm{~B}$ ). A direct comparison between the two hallucinating conditions demonstrated significant differences in activation within the bilateral insula, left middle frontal gyrus, right inferior frontal and posterior cingulate gyrus related to the somatic hallucinations and within the right superior temporal gyrus during auditory hallucinations.

\section{Discussion}

In this patient, somatic hallucinations were primarily associated with activation in the thalamus, the primary somatosensory cortex, and posterior parietal cortex, areas classically associated with tactile processing. In addition, the posterior portion of the superior temporal gyrus is also activated during normal tactile processing, and may correspond to secondary somatosensory cortex; attending to touch modulates activity in this region and in the insula bilaterally. ${ }^{13}$ Our neuroimaging findings are broadly consistent with those from an EEG study of somatic hallucinations, which associated these with activity in the postcentral gyrus, parietal operculum, insula, and the inferior parietal lobule. ${ }^{5}$ Involvement of the insula in the experience of tactile hallucinations has been shown in two cases of somatic and visceral hallucinations occurring during partial seizures caused by lesions of the insula ${ }^{14}$ and the finding that stimulation of the anterior insula in awake patients produces visceromotor and viscerosensitive responses. ${ }^{15}$

By contrast, auditory hallucinations in this patient were associated with activation in a distinct set of brain areas, particularly the right temporal cortex. Both middle and superior temporal gyri showed activation during auditory hallucinations in a group $(n=6)$ analysis using the random sampling technique described in this paper. ${ }^{1}$ The prominent involvement of the right temporal cortex may seem surprising given that the patients were perceiving speech, but is consistent with data from previous neuroimaging ${ }^{134}$ and EEG studies ${ }^{16}$ of auditory hallucinations, and with the greater right frontotemporal activation when subjects imagine another person's speech, as opposed to their own. ${ }^{17}$ Moreover, as auditory hallucinations in schizophrenia are typically derogatory and hostile in tone, the prominent engagement of right hemispheric areas might reflect processing of the prosody $^{18}$ as well as an emotional response to its content. ${ }^{19}$

A methodological limitation of this study, common to all investigations using the cognitive subtraction method, is the lack of knowledge about the resting brain activity in patients and whether it is different from resting activity in other non-hallucinating schizophrenic patients and from normal people. Similarly, although only limited conclusions can be drawn from single subject design, it is clearly advantageous to be able to map different modality hallucinations in the same subject, avoiding many of the issues in between group comparisons. A further limitation of the sampling approach is that it is less precise about the actual timing of the hallucinations, such as the duration of the preceding hallucinatory experience and reliant on the accuracy of self report.

Overall, the data from this patient suggest that hallucinations in schizophrenia are associated with activation in areas normally involved in sensory processing in that modality. This would be consistent with neuroimaging data, which suggest that visual hallucinations in patients with Charles Bonnet's syndrome are associated with activation in areas dedicated to visual processing. ${ }^{20}$

SSS is a Wellcome Trust Training Fellow. LAC is supported by the Psychiatry Research Trust. We thank the staff of the neuroimaging department at the Maudsley Hospital for their assistance in collecting the functional imaging data.

1 Shergill SS, Brammer MJ, Williams S, et al. Mapping auditory hallucinations in schizophrenia using functional magnetic resonance imaging. Arch Gen Psychiatry 2000;57:1033-8.

2 Silbersweig, DA, Stern E, Frith CD, et al. A functional neuroanatomy of hallucinations in schizophrenia. Nature 1995; 378:176-9.

3 Woodruff P, Brammer M, Mellers J, et al. Auditory hallucinations and perception of external speech. Lancet 1995; 346:1035.

4 Dierks T, Linden DEJ, Jandl M, et al. Activation of Heschl's gyrus during auditory hallucinations. Neuron 1999;22:615-

5 BaldewegT, Spence S, Hirsch SR, et al. Gamma-band electroencephalographic oscillations in a patient with somatic hallucinations. Lancet 1998;352:620-1.

6 Musalek M, Podreka HW, Suess E, et al. Regional brain function in hallucinations: a study of regional cerebral blood flow with $99 \mathrm{~m}-\mathrm{Tc}-\mathrm{HMPAO}-\mathrm{SPECT}$ in patients with auditory hallucinations, tactile hallucinations and normal controls. Compr Psychiatry 1989;30:99-108.

7 American Psychiatric Association. Diagnostic and statistical manual of mental disorders. 4th ed. Washington, DC: APA, 1994.

8 Buckner, RL. Event-related fMRI and the hemodynamic response. Hum Brain Mapp 1998;6:373-7.

9 Brammer MJ, Bullmore ET, Simmons A, et al. Generic brain activation mapping in fMRI: a non-parametric approach. Magn Reson Imaging 1997;15:763-70.

10 Bandettini PA, Jesmanowicz A, Wong EC, et al. Processing strategies for time-course data sets in functional MRI of the brain. Magn Reson Med 1993;30:161-73.

11 Talairach J, Tournoux P. Co-planar stereotactic atlas of the human brain. Stuttgart: Thieme, 1988.

12 Edgington ES. Randomisation tests. New York: Marcel Dekker, 1980

13 Johansen-Berg $\mathrm{H}$, Christensen $\mathrm{V}$, Woolrich $\mathrm{M}$, et al. Attention to touch modulates activity in both primary and secondary somatosensory areas. Neuroreport 2000;11: 1237-41.

14 Roper SN, Levesque MF, Sutherling WW, et al. Surgical treatment of epilepsy arising from the insular cortex: report treatment of epilepsy arising from the insular
of two cases. $\mathscr{f}$ Neurosurg 1993;79:266-9.

15 Ostrowsky K, Isnard J, Ryvlin P, et al. Functional mapping Ostrowsky K, Isnard J, Ryvlin P, et al. Functional mapping
of the Insula cortex: clinical implications in temporal lobe epilepsy. Epilepsia 2000;41:681-6.

16 Line P, Silberstein RB, Wright JJ, et al. Steady state visually evoked potential correlates of auditory hallucinations in schizophrenia. Neuroimage 1998;8:370-6.

7 Shergill SS, Bullmore E, Simmons A, et al. A functional MRI study of auditory verbal imagery. Psychol Med 2001;31:241-53.

18 George MS, Parekh PI, Rosinsky N, et al. Understanding emotional prosody activates right hemisphere regions. Arch Neurol 1996;53:665-70.

19 Canli T, Desmond JE, Zhao Z, et al. Hemispheric asymmetry for emotional stimuli detected with fMRI. Neuroreport 1998;9:3233-9.

20 Ffytche DH, Howard RJ, Brammer MJ, et al. The anatomy of conscious vision: an fMRI study of visual hallucinations. Nat Neurosci 1998;1:738-42. 